\title{
A new approach to hull consistency
}

\author{
LUBOMIR KOLEV \\ Technical University of Sofia \\ 1000 Sofia, Bulgaria \\ e-mail:lkolev@tu-sofia.bg
}

(Received: 10.06.2015, revised: 02.02.2016)

\begin{abstract}
Hull consistency is a known technique to improve the efficiency of iterative interval methods for solving nonlinear systems describing steady-states in various circuits. Presently, hull consistency is checked in a scalar manner, i.e. successively for each equation of the nonlinear system with respect to a single variable. In the present poster, a new more general approach to implementing hull consistency is suggested which consists in treating simultaneously several equations with respect to the same number of variables.
\end{abstract}

Key words: constraint satisfaction, hull consistency, interval methods, nonlinear circuits

\section{Introduction}

As is well known, determining all steady-states (stable and instable operating points) in nonlinear circuits (or systems) is equated to globally solving the following nonlinear system of $n$ equations in $n$ variables

$$
F(x)=0, x \in \boldsymbol{x},
$$

where $\boldsymbol{x}$ is a given initial interval domain (here and throughout the paper, bold-face letters denote interval quantities while ordinary type letters stand for real quantities). Interval methods $[1,2]$ have proved to be a reliable tool for global solution of (1). However, they suffer a serious draw-back: the computer time needed may become in some cases prohibitively long, especially for large circuits (large $n$ ) or strong nonlinearities.

One of the techniques used to improve the numerical efficiency of the interval methods is the so-called hull consistency [3]. Presently, it is applied in a scalar manner, i.e. successively to each $i$-th equation of the system with respect to a single $j$-th variable; the remaining variables are allowed to take on their initial interval values. Thus, an attempt is made to contract the initial interval $\boldsymbol{x}_{j}$ of the $j$-th variable to a narrower interval $\boldsymbol{x}_{j}^{\prime}$. If this is the case, $\boldsymbol{x}_{j}$ is replaced with $\boldsymbol{x}_{j}^{\prime}$. The same constraint satisfaction is applied to the next $(i+1)$ th equation with respect to a new variable $x_{j+1}$ trying to reduce its interval $\boldsymbol{x}_{j+1}$ to a smaller $\boldsymbol{x}_{j+1}^{\prime}$ interval.

A new more general approach is suggested here which consists in simultaneously treating several equations with respect to the same number of variables. It is expected that the con- 
traction effects of such a vector constraint satisfaction will be more pronounced than the known scalar hull consistency.

\section{The new approach}

Let $1<n^{\prime}<n$ and partition $x$ as follows: $x=(\xi, p)$ where $\xi$ is an $n^{\prime}$ dimensional auxiliary vector formed by choosing $n^{\prime}$ components $x_{j}$ of $x$ while $p$ is an $n^{\prime \prime}=n-n^{\prime}$ dimensional vector whose components are treated as parameters. To simplify the notations, it is assumed that $\xi$ is made up of the first $n^{\prime}$ components of $x$ so $p$ regroups the next $n^{\prime \prime}$ elements of $x$; also the vector $F$ is partitioned into $\left(F^{\prime}, F^{\prime \prime}\right)$ where $F^{\prime}$ involves the first $n^{\prime}$ equations of $F$. Now we consider the reduced-size nonlinear system

$$
F^{\prime}(\xi, p)=0, \xi \in \xi=\boldsymbol{x}^{\prime}, p \in \boldsymbol{p}=\boldsymbol{x}^{\prime \prime},
$$

where $\boldsymbol{\xi}$ and $\boldsymbol{p}$ correspond to the partition of $\boldsymbol{x}$ into $\boldsymbol{x}=\left(n^{\prime}, n^{\prime \prime}\right)$. Following [4 - 6], we now introduce the so-called outer solution $\boldsymbol{x}^{\text {out }}$ of the nonlinear parameter system (2). If the section

$$
\boldsymbol{S}_{j}=\boldsymbol{x}_{j}^{\text {out }} \cap \boldsymbol{x}_{j}^{\prime}
$$

is smaller than the corresponding $\boldsymbol{x}_{j}^{\prime}$, then $\boldsymbol{x}_{j}^{\prime}$ is replaced by $\boldsymbol{S}_{j}$ Since $n_{j}^{\prime}>1$, system (2) imposes more stringent constraints than a single equation $F_{i}(x)=0$ with respect to a single variable $x_{j}$ so it is expected that $\boldsymbol{S}_{j}$ will, in general, be a better contraction than the scalar contraction. The reduced intervals $\boldsymbol{S}_{j}$ are now substituted for the corresponding initial intervals $\boldsymbol{x}_{j}^{\prime}$ in (2) and a new system (2) is formed where $\xi$ and $p$ regroup the next $n^{\prime}$ components of $x$ and $F$, respectively. This process may continue until the reduction of the intervals is considered sufficient or some intersection $\boldsymbol{S}_{j}$ between $\boldsymbol{x}_{j}^{\text {out }}$ and $\boldsymbol{x}_{j}^{\prime}$ becomes empty. In the latter case, the system of constraints is inconsistent.

From the view point of the new vector approach, the scalar hull consistency reduces to the special case where $n^{\prime}=1$.

It is seen that the core of the vector approach to hull consistency is the determination of the outer solution $\boldsymbol{x}^{\text {out }}$ of (2). The latter task is solved in two stages:

(i) the nonlinear system (2) is transformed to a linear interval parametric (LIP) system [7 - 10]

$$
A(q, p) z=b(p), q \in \boldsymbol{x}, p \in \boldsymbol{p},
$$

(ii) now an outer solution $\boldsymbol{x}^{b}$ of (3) is found.

Finally, the $\boldsymbol{x}^{\text {out }}$ is given approximately by $\boldsymbol{x}^{b}$.

\section{Transformation to a LIP system}

System (2) is written as

$$
f(x, p)=0, \quad p \in \boldsymbol{p} \subset R^{m},
$$




$$
x \in \boldsymbol{x}^{0} \subset R^{n^{\prime}} .
$$

The solution set of $f$ is the set

$$
S_{f}(\boldsymbol{p}):=\{x: f(x, p)=0, p \in \boldsymbol{p}\} .
$$

Its interval hull will be denoted $\boldsymbol{x}^{*}$; any other interval $\boldsymbol{x}^{b}$ containing $\boldsymbol{x}^{*}$ is referred to as an outer interval solution of (4a).

Two methods for transforming the nonlinear system (4) to a LIP system (3) will be suggested here. We first present the most popular approach among the known methods.

\subsection{Standard approach}

It is based on the interval version of the Newton method [3]. Now (4a) is replaced with

$$
f(y, p) \in f(x)+\boldsymbol{J}(\boldsymbol{x}, \boldsymbol{p})(y-x),
$$

which becomes

$$
\boldsymbol{J}(\boldsymbol{x}, \boldsymbol{p})(y-x)=-f(x, p)
$$

if $(y, p)$ is a zero of $f$.

A better approach is possible based on a parametric form of the interval Newton method.

\subsection{First method}

The first possibility is to transform the original nonlinear system (4) into an equivalent linear interval parametric (LIP) system. The novel approach will be initially presented for a non-parametric system

$$
f(x)=0, x \in \boldsymbol{x} .
$$

It is based on the use of the slope matrix $S(y, x)$ and the equality

$$
f(y)=f(x)+S(y, x)(y-x),
$$

where $y$ and $x$ have some fixed values (typically, $x$ is the center $x^{c}$ of $\boldsymbol{x}$ ). We "free" the components $y_{k}$ of $y$ and consider them as components of a parameter vector $p$, i.e.

$$
p=\left(y_{1}, \ldots, y_{n}\right) \in \boldsymbol{x}=\left(\boldsymbol{x}_{1}, \ldots, \boldsymbol{x}_{n}\right) .
$$

Let

$$
a_{i j}(p)=S_{i j}(p, x) \text {, }
$$

be the entries of the parametric matrix $A(p)$. On account of (7) to (9)

$$
f(y) \in f(x)+A(p)(y-x), \quad p \in \boldsymbol{x} .
$$


The right-hand side of (10) is the known linear parametric approximation (LPA) of $f$ in $x$ [7]. If $y$ is a zero of $f$

$$
A(p)(y-x)=-f(x), \quad p \in \boldsymbol{x},
$$

or, equivalently

$$
A(p) z=b(x), \quad p \in \boldsymbol{x} .
$$

This approach has been generalized to the case of the parametric system (3) in [10]. Now, for a fixed $p$ formula (7) becomes

$$
f(y, p)=f(x, p)+S(y, x, p)(y-x),
$$

where most often $x=x^{c}$. Once again, we "free" the components $y_{k}$ of $y$ to take on values in $\boldsymbol{x}$. Thus, we introduce the additional parameter vector

$$
q=\left(y_{1}, \ldots, y_{n}\right) \in \boldsymbol{x}=\left(\boldsymbol{x}_{1}, \ldots, \boldsymbol{x}_{n}\right),
$$

and let

$$
a_{i j}(q, p)=S_{i j}(q, x, p),
$$

be the entries of the parametric matrix $A(q, p), q \in \boldsymbol{x}, p \in \boldsymbol{p}$ ( $x$ is fixed). For each $p \in \boldsymbol{p}$

$$
f(y, p) \in f(x)+A(q, p)(y-x), q \in \boldsymbol{x} \quad p \in \boldsymbol{p} .
$$

If $(y, p)$ is a zero of $f$

$$
A(q, p) z=b(p), q \in \boldsymbol{x}, p \in \boldsymbol{p},
$$

where $z=y-x$ and $b(p)=-f(x, p)$. The linear parametric system (15) is the novel LPA model suggested here to tackle the problem of obtaining an outer approximation of the solution set of (4a). It is argued that (15) is a better model that (5) to bound the solutions of (4a). Indeed, consider the sets

$$
\begin{gathered}
S_{J P}=\{z: J z=b, J \in \boldsymbol{J}(\boldsymbol{x}, \boldsymbol{p})\}, \\
S_{p q}=\{z: \quad A(q, p) z=b(p), \quad q \in \boldsymbol{x}, \quad p \in \boldsymbol{p}\} .
\end{gathered}
$$

Clearly,

$$
S_{p q} \subset S_{J P}
$$

since $\boldsymbol{J}(\boldsymbol{x}, \boldsymbol{p})$ has $n^{2}$ independent entries, each being an interval extension of the function $J_{i j}(x, p)$ of $n+m$ arguments, while there are only $n+m$ independent elements in $A(q, p)$ and $m$ independent elements in $b(p)$. If $Z_{J P}^{*}, Z_{p q}^{*}$ denote the interval hulls of $S_{Y P}, S_{p q}$, respectively, while $\boldsymbol{Z}_{J P}$ and $\boldsymbol{Z}_{p q}$ are some outer enclosures, then

$$
Z_{p q}^{*} \subset Z_{j p}^{*},
$$


and hopefully

$$
Z_{p q} \subset Z_{J P},
$$

(unlike (19a), (19b) is not guaranteed: it all depends on the efficiency of the methods used to compute $\boldsymbol{Z}_{J P}$ and $\boldsymbol{Z}_{p q}$ ). Nowadays, there exist various methods [4, 5, 8] for computing $Z_{p q}^{*}$ and $\boldsymbol{Z}_{p q}$ (see [11] for a list of references).

\subsection{Second method}

It is based on the use of the Hansen-Sengupta operator [3] in parametric form. The approach of $\S 3.2$ is applicable only if the slope matrix $S(q, x, p)$ is available in analytical form. If this is not the case, then the Jacobian matrix in parametric form $J(q, p)$ can be used as suggested in [11]. Thus, (13a) is replaced with

$$
a_{i j}(q, p)=J_{i j}\left(q_{1}, \ldots, q_{n}, p_{1}, \ldots, p_{m}\right), \quad q_{k} \in \boldsymbol{x}_{k}, k=1, \ldots, n, \quad p_{k} \in \boldsymbol{p}_{k}, k=1, \ldots, m,
$$

It is seen that each element $a_{i j}(q, p)$ depends on all $n+m$ parameters $q_{k}$ and $p_{k}$.

A better LPA is possible which is based again on the Hansen-Sengupta operator. In its standard (non-parametric) form, it encloses each function $f_{i}(y)$ by the following expression

$$
f_{i}(y) \in f_{i}(x)+\sum_{j=1}^{n}\left(y_{j}-x_{j}\right) g_{i j}\left(\boldsymbol{x}_{1}, \ldots, \boldsymbol{x}_{j} ; x_{j+1}, \ldots, x_{n}\right),
$$

where $x_{j+1}, \ldots, x_{n}$ are real numbers. We now write (21) in a parametric form

$$
f_{i}(y) \in f_{i}(x)+\sum_{j=1}^{n}\left(y_{j}-x_{j}\right) g_{i j}\left(q_{1}, \ldots, q_{j} ; x_{j+1}, \ldots, x_{n}\right), \quad \boldsymbol{q}_{1} \in \boldsymbol{x}_{1}, \ldots, q_{1} \in \boldsymbol{x}_{j} .
$$

Hence, using the Hansen-Sengupta operator, we introduce a parametric matrix $A(q)$ with elements

$$
a_{i j}(q)=g_{i j}\left(q_{1}, \ldots, q_{j} ; x_{j+1}, \ldots, x_{n}\right), \quad \boldsymbol{q}_{1} \in \boldsymbol{x}_{1}, \ldots, q_{j} \in \boldsymbol{x}_{j} .
$$

It is seen that now fractions $(1 / 2)(1-1 / n)$ in (22) are real parameters. This determines the better performance of methods based on the Hansen-Sengupta LPA as compared to those using the Jacobian LPA since they lead to tighter outer solutions.

On account of the foregoing, if $S(q, p)$ cannot be found in analytical form, the HansenSengupta form $g(q, p)$, should be used rather than $J(q, p)$. In that case, the elements of the parametric matrix $A(q, p)$ are given not by (20) but as follows:

$$
a_{i j}(q, p)=J_{i j}\left(q_{1}, \ldots, q_{j}, p_{1}, \ldots, p_{m}\right), q_{k} \in \boldsymbol{x}_{k}, k=1, \ldots j, \quad p_{k} \in \boldsymbol{p}_{k}, k=1, \ldots, m .
$$

Yet another still better option is to apply the Hansen-Sengupta approach to the whole vector $u(q, p)$ which leads to a parametric matrix $A(u)$ with components: 


$$
a_{i j}(u)=g_{i j}\left(u_{1}, \ldots, u_{j} ; u_{j+1}^{0}, \ldots, u_{n+m}^{0}\right), \quad u_{k} \in \boldsymbol{u}_{k}, k=1, \ldots, j,
$$

having the least possible number of interval entries.

\section{Outer interval parametric solution}

\subsection{Standard approach}

So far, we have consider the problem of choosing an appropriate LPA model (13a), (15) or (23) of system (4). Now we proceed to presenting a new method for determining a tight outer interval (OI) solution of the selected LPI model which is written for simplicity in the form

$$
A(p) x=b(p), p \in \boldsymbol{p},
$$

( $p$ stands for $(q, p)$ ). The new method is based on the use of a so-called $p$-solution $\boldsymbol{x}(p)$ of (24) (recently introduced in [11]). The $p$-solution (parameterized solution) is given by the linear interval form (LIF)

$$
\boldsymbol{x}(p)=L p+\boldsymbol{a}, p \in \boldsymbol{p},
$$

where $L$ is a real $n \times m$ matrix while $\boldsymbol{a}$ is an $n$-dimensional interval vector.

Remark. The solution $\boldsymbol{x}(p)$ can be found by a method given in [11] which is applicable for the case where the components of $A(p)$ and $b(p)$ are linear functions of the parameter entries. This is, however, not a restriction since the nonlinear $A(p)$ and $b(p)$ can be always reduced to linear functions with additional parameters using a well-known technique [7].

It turns out that an alternative nonlinear form $p$-solution can be constructed whose $i$ th component

$$
x_{i}(p)=p^{T} Q_{i} p+L_{i} p+c_{i}, p \in \boldsymbol{p}
$$

is given by the quadratic interval (QI) form given in the right - hand side of (26). For relatively narrow $\boldsymbol{p}$, the nonlinear $p$ - solution will be better than its linear counterpart.

\subsection{Quadratic iterative method}

In this subsection, an iterative method for determining a quadratic parametric (QP) solution $\boldsymbol{x}(p)$ will be suggested which is based on the QI form (26), from one side, and the following iterative process. The linear model (24) is written equivalently in the form

$$
\left(A^{(0)}-\sum_{\mu} p_{\mu} A^{(\mu)}\right) x(p)=b^{0}+B p,
$$

which is transformed into the fixed-point representation

$$
x(p)=(I-A(p)) x(p)+B p, \quad p \in \boldsymbol{p} .
$$


On the account of (27), the iterative process is

$$
x^{(\kappa+1)}(p)=\left(A^{(0)}-\sum_{\mu} p_{\mu} A^{(\mu)}\right) x^{(\kappa)}(p)+b^{0}+B p, \quad \kappa \geq 0, x^{(0)}=x^{0},
$$

where $A^{(0)}=I-A^{0}$ and (assuming $A^{0}$ nonsingular) $x^{0}$ is the solution of (24) for $p=0$. The new method will be referred to as QIF method.

Initially, the QIF method repeats the first steps $\kappa=0$ and $\kappa=1$ until the quadratic representation (26) is formed. At that point, the quadratic part is, however, not literalized so the next step $\kappa=2$ is started with

$$
x_{i}^{(2)}(p)=p^{T} Q_{i}^{(2)} p+L_{i}^{(2)} p+c_{i}^{(2)} .
$$

We now use the relationship

$$
x^{(3)}(p)=T(p) x^{(2)}(p)+b^{0}+B^{0} p=f^{2}(p),
$$

where $T(p)$ is the bracketed expression in (28). As can be easily seen, $f_{i}^{(2)}$ in (30) is a cubic expression which is written in the form

$$
f_{i}^{(2)}(p)=\sum_{j} C_{i j}\left(p_{j}\right)+R_{i j}(p),
$$

where $C_{j j}\left(p_{j}\right)$ is a cubic function of one variable while $R_{j j}(p)$ regroups all the other terms. Now each $j$ th cubic term is approximated outwardly by a corresponding quadratic interval form so we get

$$
\boldsymbol{q}_{i}^{(3)}(p)=p^{T} Q_{j}^{(3)} p+L_{i}^{(3)} p+c_{i}^{(3)}+\boldsymbol{s}_{j}^{(3)} .
$$

At the next step for $\kappa=3$, the relationship

$$
x^{(4)}(p)=T(p) x^{(3)}(p)+b^{0}+B^{0} p
$$

is replaced with

$$
x^{(4)}(p)=T(p) \boldsymbol{q}^{(3)}(p)+b^{0}+B^{0} p=f^{(3)}(p) .
$$

Now $f^{(3)}(p)$ contains a cubic function which is again approximated in a quadratic manner. Evidently, this process of successively generating new cubic functions and their subsequent quadratic approximation can continue for $\kappa \geq 3$.

Consider the range $\boldsymbol{q}^{(\kappa)}(\boldsymbol{p})$ of the corresponding QI form $\boldsymbol{q}^{(\kappa)}(p)$. Clearly, $\boldsymbol{q}^{(\kappa)}(\boldsymbol{p})$ is an interval vector having the properties

$$
S^{(k+1)} \subset \boldsymbol{q}^{(\kappa)}(\boldsymbol{p}),
$$

where $S^{(k+1)}$ is the image of $f^{(k)}$ in $\boldsymbol{p}$ and 


$$
\boldsymbol{q}^{(k)}(\boldsymbol{p}) \subset \boldsymbol{q}^{(\kappa+1)}(\boldsymbol{p}) .
$$

The distance between two such interval vectors in (36) will be assessed using

$$
q(\boldsymbol{a}, \boldsymbol{b})=\max \left\{\max _{i}\left|\underline{a}_{i}-\underline{b}_{i}\right|, \max _{i}\left|\bar{a}_{i}-\overline{b_{i}}\right|\right\} .
$$

Now we can formulate the main result of the subsection.

Theorem 1 Assume that the matrix $A^{0}$ is non-singular and the sequence $\left\{\boldsymbol{q}^{(\propto)}(\boldsymbol{p})\right\}, \kappa \geq 1$ is convergent in the sense of (37) to a limit $\boldsymbol{q}^{(\propto)}(\boldsymbol{p})$. Then:

(i) the interval vector

$$
\boldsymbol{x}=\boldsymbol{q}^{(\infty)}(\boldsymbol{p})
$$

is an OI solution to (1);

(ii) the quadratic interval form

$$
\boldsymbol{x}(p)=\boldsymbol{q}^{(\infty)}(p)=c^{(\infty)}+L^{(\infty)} p+Q^{(\infty)} \varphi(p)+\boldsymbol{s}^{(\infty)},
$$

determines a $Q P$ solution to (3);

(iii) the matrix $A(p)$ is non-singular for each $p \in \boldsymbol{p}$.

On account of Theorem 1 , the outer solution $\boldsymbol{x}^{b}$ sought is given by $\boldsymbol{x}^{(k+1)}$. The above method for determining the outer interval (OI) solution $\boldsymbol{x}^{b}$ of (24) and, hence, of any of the LPA models (13a), (15) or (23) of system (4), using the quadratic parametric (QP) form (39) will be referred to as the OIQP method.

\section{Interval hull solution}

A narrower interval than $\boldsymbol{x}^{b}$ is the interval hull (IH) solution $\boldsymbol{x}^{*}$ of (11). A method for determining $\boldsymbol{x}^{*}$ has been proposed in [6] which is based on the linear LP-solution (25). An improved version that employs the quadratic $p$-solution (26) is suggested here.

As in [11], $\boldsymbol{x}^{*}$ is determined componentwise by computing each $\boldsymbol{x}_{k}^{*}=\left[l_{k}^{*}, u_{k}^{*}\right]$ using an appropriate modification of the method in [11]. We bound ourselves to the lower end of $l_{k}^{*}$.

Determination of $l_{k}^{*}$

The value of $l_{k}^{*}$ is found as the global solution of the following optimization problem:

$$
\underline{l}_{k}^{*}=\min e_{k}^{T} x
$$

subject to the constraint

$$
A(p) x=b(p), \quad p \in \boldsymbol{p} .
$$

The computational scheme for solving (40) involves two basic phases at each iteration: (i) find in $\boldsymbol{p}$ an upper bound $l_{k}^{u}$ on $l_{k}^{*}$, (ii) using $l_{k}^{u}$ and a related constraint equation, try to re- 
duce the current domain $\boldsymbol{p}$ to a narrower domain $\boldsymbol{p}^{\prime}$ applying some constraint satisfaction technique. The iterations continue until the width of the current domain becomes smaller than a given threshold $\varepsilon_{p}$.

In [11], the above two phases were both implemented using the corresponding LP solution which results in a linear constraint equation. We now show that the efficiency of the method based on the associated iteration (28) can be improved by resorting to the related QP solution. Indeed, as can be easily seen, the constraint equation is now nonlinear.

On account of (39) (dropping the superscript ( $\infty$ )) the constraint is

$$
\boldsymbol{q}(p)=Q \phi(p)+L p+\boldsymbol{a}, p \in \boldsymbol{p}, \quad \phi_{j}(p)=\phi_{j}\left(p_{j}\right)=p_{j}^{2},
$$

where $Q$ is a three-dimentional $n \times m \times m$ array, $L$ and $\boldsymbol{a}$ having the same meaning (but different entries) as in (25). Now (41) is rewritten as

$$
Q_{k j_{0} p} p_{j_{0}}^{2}+L_{k j_{0}} p_{j_{0}}+\boldsymbol{R}_{k}=0, \quad p_{j_{0}} \in \boldsymbol{p}_{j_{0}},
$$

where $j_{0}$ is a chosen index, $\boldsymbol{R}_{k}$ is an interval combining the interval extensions of the remaining terms for $j \neq j_{0}$. Equation (42) is put in the form $p_{j_{0}}+2 b+c=0$ so

$$
p_{j_{0}}^{(1)}=-b-\sqrt{b^{2}-c}, \quad p_{j_{0}}^{(2)}=-b+\sqrt{b^{2}-c},
$$

if

$$
c<b^{2} .
$$

This approach offers better possibilities to contract the current $\boldsymbol{p}$ as compared to the known linear constraint technique. Indeed, now an additional contracting effect appears whenever (44) is violated since $c$ involves a sum of quadratic expressions $\alpha_{j} \varphi_{j}\left(p_{j}\right), j \neq j_{0}$. The resulting method for determining the interval hull $(\mathrm{IH})$ solution using the quadratic parametric $(\mathrm{QP})$ form will be referred to as the IHQP method.

\section{Conclusions}

A new vector approach to hull consistency has been suggested which reduces, essentially, to determining a tight outer solution $\boldsymbol{x}^{\text {out }}$ of the nonlinear parametric system (4). To this end, system (4) is first transformed to a corresponding linear parametric model in form of systems (13a), (15) or (23). Then a tight outer solution $\boldsymbol{x}^{b}$ of the linear system chosen is computed, using the OIQP method (employing the quadratic parametric form (39) and based on Theorem 1). A better but more expensive choice is to compute the interval hull solution $\boldsymbol{x}^{*}$ using the IHQP method.

Future research should concentrate on the numerical implementation of the new vector form hull consistency, confirming experimentally its improved efficiency as compared to the known scalar form hull consistency. 


\section{Acknowledgement}

The author is grateful to the anonymous reviewers for their variable remarks.

\section{References}

[1] Kolev L., Interval methods for circuit analysis, World Scientific (1993).

[2] Kolev L., An iterval method for global nonlinear analysis, IEEE Transactions on Circuits and Systems-I (47): 675-683 (2000).

[3] Hansen E., Walster G., Global optimization using interval analysis, Marcel Dekker (2004).

[4] Popova E., Generalization of the parametric fixed-point iteration, Proceedings in Applied Mathematics and Mechanics (4): 680-681 (2004).

[5] Skalna I., A method for outer interval solution of parameterized systems of linear equations, Reliable Computing (12): 107-120 (2006).

[6] Kolev L., Eigenvalue range determination for interval and parametric matrices, International Journal of Circuit Theory and Applications (38): 1027-1061 (2010).

[7] Kolev L., Some ideas towards global optimization of improved efficiency, Global Optimization Integrating Convexity, Optimization, Logic Programming and Computational Algebraic Geometry workshop, 4-9 November, Wien, pp. 1-39 (2006).

[8] Neumaier A., Pownuk A., Linear systems with large uncertainties, with applications to truss structures, Reliable Computing (13): 149-172 (2007).

[9] Kolev L., Determining the stability margin in linear interval parameter electric circuits via a DAE model, International Journal of Circuit Theory and Applications (40): 903-926 (2012).

[10] Kolev L., Global solution of a class of interval parameter optimization problems, Proceedings of the XXVII International Symposium on Theoretical Electrical Engineering, June 24-26, Pilsen, II 11-12 (2013).

[11] Kolev L., Parameterized solution of linear interval parametric systems, Applied Mathematics and Computation (246): 229-246 (2014). 